\title{
Effective Action and Schwinger Pair Production in Scalar QED
}

\author{
Sang Pyo Kin* \\ Department of Physics, Kunsan National University, Kunsan 573-701, Korea and \\ Asia Pacific Center for Theoretical Physics, Pohang 790-784, Korea \\ Hyun Kyu Leđ丹 \\ Department of Physics and BK21 Division of Advanced Research and Education in Physics, Hanyang University, Seoul 133-792
} (Dated:)

\begin{abstract}
Some astrophysical objects are supposed to have very strong electromagnetic fields above the critical strength. Quantum fluctuations due to strong electromagnetic fields modify the Maxwell theory and particularly electric fields make the vacuum unstable against pair production of charged particles. We study the strong field effect such as the effective action and the Schwinger pair production in scalar QED.

Keywords: Strong QED, Effective action, Schwinger pair production, Astrophysical objects with strong electromagnetic fields
\end{abstract}

PACS numbers: 12.20.-m, 13.40.-f, 11.10.Wx, 11.15.Tk

\section{INTRODUCTION}

Gravitational interaction and electromagnetic interaction are the most important fundamental interactions in astrophysics. The non-relativistic Newtonian gravity is no longer a proper theory for strong gravity. The Einstein gravity, a new paradigm, replaces the Newtonian gravity and predicts entirely new phenomena such as black holes when the gravitational interaction is extremely strong. One may wonder how the Maxwell theory would be modified for extremely strong sources of charges and current or external electromagnetic fields. As the Maxwell theory is already a relativistic theory for electromagnetic phenomena, it is not likely that a new theory would replace the Maxwell theory even for reasonably strong sources or electromagnetic fields up to the unification scale for gravity and electromagnetism. Thus, below the unification scale, the Maxwell theory is still the building block for quantum phenomena.

Virtual pairs of charged particles experience the interaction with the background of strong electromagnetic fields and undergo quantum fluctuations contributing to the effective action. One remarkable phenomenon due to a strong electromagnetic field, in particular, strong electric field, is Schwinger pair production of charged particles from the vacuum [1]. In fact, the virtual particle and antiparticle from the Dirac sea can gain a sufficient kinetic energy comparable or greater than the rest mass energy over the Compton wavelength and then tunnel the potential barrier from the Dirac sea to substantiate into real pairs. The critical strength for electron-positron pair production is $E_{c}=m_{e}^{2} c^{3} / e \hbar\left(1.3 \times 10^{16} \mathrm{~V} / \mathrm{cm}\right)$.

A particle with charge $q$ and mass $m$ in a constant magnetic field undergoes circular motions with the Lar-

\footnotetext{
*Electronic address: sangkim@kunsan.ac.kr
}

${ }^{\dagger}$ Electronic address: hyunkyu@hanyang.ac.kr mor frequency $\omega_{c}=q B / m c$. In quantum theory charged particles occupy the Landau levels with energy $E=$ $\hbar \omega_{c} n$ and in a strong magnetic field of $B_{c}=m^{2} c^{2} / q \hbar$ $\left(4.4 \times 10^{13} \mathrm{G}\right.$ for an electron), the energy difference of Landau levels $\delta E=\hbar \omega_{c}$ can be comparable to the rest mass of the particle. In the transverse direction of a magnetic field above the critical strength electrons of an atom are strongly bounded and fill the lowest Landau levels but in the parallel direction are attracted by the Coulomb force. Further, the energy of Landau levels yields a quantum correction to the Maxwell term. It turns out that the quantum correction contributes nonlinear terms of Lorentz invariant field tensors to the effective action and the vacuum thus becomes polarized effectively similar to nonlinear polarized media [1, 2, 3] (For a review on strong QED, see [4]).

At present the most strong electromagnetic fields are generated not by terrestrial experiments but by astrophysical sources. Even the strong electromagnetic fields that would be generated by terrestrial experiments or International Linear Collider in the future [5] are still below the magnetic fields of neutron stars, which are remnants of massive stars. The magnetic flux of a normal star with radius $R_{0}$ and field $B_{0}$ is frozen during the collapse and amplified to $B=B_{0}\left(R_{0} / R_{n}\right)^{2}$. The magnetic field may be further amplified by dynamos. Neutron stars have magnetic fields ranging from $10^{8} \mathrm{G}$ to $10^{15} \mathrm{G}[\underline{6}$. . Magnetars have the strongest magnetic field above the critical strength. The electromagnetic phenomena in magnetars would significantly differ from the Maxwell theory due to QED effects [7, 8].

Only with electromagnetic interactions it is hard to accumulate charges to generate an electric field beyond the critical strength partly because of the repulsive force among the same kind of charges and partly because of the decay of the field itself by emitting pairs. However, strange stars, hypothetical quark stars of quark matter 9, 10, 11, 12], can hold electrons on the crust by attractive Coulomb force by positively charged core which in 
turn tightly bounded by nuclear force [13, 14]. The electrosphere of strange stars, if exist, can be the strongest source for electric fields with two order higher than the critical strength and the emission of charged particles from Schwinger pair production may be an evidence of strange stars [15, 16, 17].

The purpose of this paper is to study the effective action in scalar QED in the presence of a strong external electromagnetic field and the Schwinger pair production due to the vacuum instability. Here we present a novel method to calculate the effective action particularly in electric fields. In the Hamiltonian approach developed in Ref. [18], which is based on the invariant method [19], the evolution operator can be expressed by a two-mode squeezed operator, in terms of which the ingoing vacuum evolves to the outgoing vacuum. The scattering amplitude of the ingoing vacuum to the outgoing vacuum is the expectation value of the two-mode squeeze operator and the effective action is thus determined by the Bogoliubov coefficients between the ingoing and outgoing particle and antiparticle operators. The adiabaticity of the evolution assumed in Ref. [20] is not required for the effective action. Further we find the Fock space of number states of particles and antiparticles, which evolve from the ingoing vacuum to the outgoing vacuum.

The organization of this paper is as follows. In Sec. II, we briefly review the effective action in scalar QED under the influence of uniform magnetic field and electric field. In Sec. III, we apply the Hamiltonian approach to study the Fock space for charged particles and antiparticles under an external electromagnetic field. In Sec. IV, we find the effective action in terms of the Bogoliubov coefficients by calculating the scattering amplitude of the ingoing vacuum to the outgoing vacuum.

\section{EFFECTIVE ACTION IN CONSTANT B AND E}

In this section, following the Hamiltonian approach developed in Ref. [18], we study scalar QED described by the Klein-Gordon equation for a charged boson with $q(q>0)$ and $m$ (in units with $\hbar=c=1$ and with metric signature $(+,-,-,-))$

$$
\left[\eta^{\mu \nu}\left(\partial_{\mu}+i q A_{\mu}\right)\left(\partial_{\nu}+i q A_{\nu}\right)+m^{2}\right] \phi(\mathbf{x}, t)=0 .
$$

The charged boson has the Lagrangian

$$
L=\int d^{3} x\left[\eta^{\mu \nu}\left(\partial_{\mu}-i q A_{\mu}\right) \phi^{*}\left(\partial_{\mu}+i q A_{\mu}\right) \phi-m^{2} \phi^{*} \phi\right] .
$$

In a constant magnetic field along the $z$-direction, we choose the gauge in a symmetric form

$$
A_{\mu}=\left(0,-\frac{B y}{2}, \frac{B x}{2}, 0\right) \text {. }
$$

As classically the charged particle moves along a spiral of circular motion in the transverse direction and linear motion along the parallel direction of the magnetic field, we decompose the field $\phi=\phi_{\perp}(x, y) \phi_{z}$, with the transverse part being real and the parallel part being complex. After integrating over the transverse direction, the corresponding Hamiltonian is given by

$H=\int d z\left[\pi^{*} \pi+\left(\partial_{z} \phi_{z}^{*}\right)\left(\partial_{z} \phi_{z}\right)+\left(H_{\perp}(x, y)+m^{2}\right) \phi_{z}^{*} \phi_{z}\right]$,

where $\pi=\dot{\phi}^{*}$ and $\pi^{*}=\dot{\phi}$ are the conjugate momenta, and the transverse part is

$$
H_{\perp}(x, y)=p_{x}^{2}+p_{y}^{2}+\left(\frac{q B}{2}\right)^{2}\left(x^{2}+y^{2}\right)+(q B) L_{z} .
$$

Here $p_{x}=-i \partial_{x}, p_{y}=-i \partial_{y}$ and $L_{z}=x p_{y}-y p_{x}$ is the $z$-component of angular momentum. The KleinGordon equation has the solution of the form $\phi(\mathbf{x}, t)=$ $e^{-i \omega t+i k_{z} z} \phi_{\perp}(x, y)$, where

$$
\left[H_{\perp}(x, y)+m^{2}+k_{z}^{2}\right] \phi_{\perp}(x, y)=\omega^{2} \phi_{\perp}(x, y)
$$

Note that the transverse part is a two-dimensional oscillator with the mass $1 / 2$ and the frequency $q B$ together with the $z$-component of angular momentum.

One may use the oscillator representation of $U(3)$, which has $S O(3)$ as a subgroup, with the generators [21]

$$
T_{i j}=\frac{1}{2}\left(c_{i}^{\dagger} c_{j}+c_{j} c_{i}^{\dagger}\right)
$$

where $c_{i}$ and $c_{i}^{\dagger}$ are annihilation and creation operators for the component $i=x, y, z$. It follows that the angular momentum takes the form

$$
L_{z}=i\left(T_{y x}-T_{x y}\right),
$$

and the transverse part thus has the oscillator representation

$$
H_{\perp}=q B\left(T_{x x}+T_{y y}\right)+i(q B)\left(T_{y x}-T_{x y}\right) .
$$

In the new basis

$$
c_{ \pm}=\frac{1}{\sqrt{2}}\left(c_{x} \pm i c_{y}\right)
$$

the transverse part takes the diagonal form

$$
\begin{aligned}
H_{\perp} & =q B\left(T_{++}+T_{--}\right)+q B\left(T_{--}-T_{++}\right) \\
& =2(q B) T_{--} .
\end{aligned}
$$

The eigenstates of the transverse part are the number states $\left|n_{-}\right\rangle=\left(c_{-}^{\dagger}\right)^{n_{-}}\left|0_{-}\right\rangle / \sqrt{n_{-} !}$with the energy eigenvalue

$$
H_{\perp}\left|n_{-}\right\rangle=q B\left(2 n_{-}+1\right)\left|n_{-}\right\rangle .
$$

Hence the energy eigenstate and eigenvalue of the Hamiltonian (4) are given by

$$
\begin{aligned}
H\left|n, k_{z}\right\rangle & =\omega\left(n, k_{z}\right)\left|n, k_{z}\right\rangle \\
& =\sqrt{m^{2}+k_{z}^{2}+q B(2 n+1)}\left|n, k_{z}\right\rangle .
\end{aligned}
$$


The effective action of scalar QED may be found from the scattering amplitude

$$
\left.e^{i S_{\text {eff }}}=\langle\text { out }| \text { in }\right\rangle,
$$

where the outgoing state evolves from the ingoing state as

$$
\mid \text { out }\rangle=U \mid \text { in }\rangle \text {. }
$$

Using the evolution operator for the Hamiltonian (4) in the magnetic field

$$
U=e^{-i H t}
$$

we find the effective action

$$
e^{i S_{\mathrm{eff}}}=\prod_{n, k_{z}} e^{i \omega\left(n, k_{z}\right)}\left\langle n, k_{z} \mid n, k_{z}\right\rangle
$$

Now the effective action per unit time and unit area, $\mathcal{L}_{\text {eff }}=S_{\text {eff }} /($ time $) \times($ area $)$, is given by

$$
\mathcal{L}_{\mathrm{eff}}=\frac{q B}{(2 \pi)^{2}} \int_{-\infty}^{\infty} d k_{z} \sum_{n=0}^{\infty} \sqrt{m^{2}+k_{z}^{2}+q B(2 n+1)}
$$

where $q B /(2 \pi)$ is the number of Landau levels and $1 /(2 \pi)$ is the number of states for $k_{z}$. To perform the summation and integral, following Ref. [22], we differentiate (18) twice with respect to $m^{2}$ and sum over $n$ to obtain

$$
\frac{\delta^{2} \mathcal{L}_{\mathrm{eff}}}{\delta\left(m^{2}\right)^{2}}=\frac{q B}{16 \pi^{2}} \int_{0}^{\infty} d s e^{-m^{2} s} \frac{1}{\sinh (q B s)} .
$$

Integrating twice with respect to $\mathrm{m}^{2}$, we obtain the effective action

$$
\begin{aligned}
\mathcal{L}_{\text {eff }}(B)=- & \frac{1}{16 \pi^{2}} \int_{0}^{\infty} d s \frac{e^{-m^{2} s}}{s^{3}} \\
& \times\left[\frac{q B s}{\sinh (q B s)}-1+\frac{(q B s)^{2}}{6}\right],
\end{aligned}
$$

where we fix the integration constants such that $\mathcal{L}_{\text {eff }}(B=$ $0)=0$. The effective action for a constant electric field may be obtained from the duality $B=i E$

$$
\begin{aligned}
\mathcal{L}_{\text {eff }}(E)=- & \frac{1}{16 \pi^{2}} \int_{0}^{\infty} d s \frac{e^{-m^{2} s}}{s^{3}} \\
& \times\left[\frac{q E s}{\sin (q E s)}-1-\frac{(q E s)^{2}}{6}\right] .
\end{aligned}
$$

In terms of the Lorentz invariant tensors

$$
\begin{aligned}
\mathcal{F} & =\frac{1}{4} F_{\mu \nu} F^{\mu \nu}=-\frac{1}{2}\left(\mathbf{E}^{2}-\mathbf{B}^{2}\right)=\frac{1}{2}\left(X_{r}^{2}-X_{i}^{2}\right), \\
\mathcal{G} & =\frac{1}{4} F_{\mu \nu} \tilde{F}^{\mu \nu}=-\mathbf{E} \cdot \mathbf{B}=X_{r} X_{i},
\end{aligned}
$$

the effective action can be expressed as

$$
\begin{aligned}
& \mathcal{L}_{\text {eff }}(B, E)=-\frac{1}{16 \pi^{2}} \int_{0}^{\infty} d s \frac{e^{-m^{2} s}}{s^{3}} \\
& \quad \times\left[\frac{\left(q X_{r} s\right)\left(q X_{i} s\right)}{\sinh \left(q X_{r} s\right) \sin \left(q X_{i} s\right)}-1-\frac{(q s)^{2}}{6}\left(X_{r}^{2}-X_{i}^{2}\right)\right]
\end{aligned}
$$

For another derivation of the effective action, see Refs. [1, 2, 3].

The real part of the effective action up to quartic power of fields is

$$
\operatorname{Re} \mathcal{L}_{\text {eff }}(B, E)=\frac{q^{4}}{720 \pi^{2} m^{4}}\left(4 \mathcal{F}^{2}+7 \mathcal{G}^{2}\right) .
$$

The imaginary part comes from the pole structure of the integral and takes the form

$$
2 \operatorname{Im} \mathcal{L}_{\mathrm{eff}}(B, E)=\frac{1}{8 \pi^{3}} \sum_{n=1}^{\infty}(-1)^{n+1}\left(\frac{q X_{i}}{n}\right)^{2} e^{-\frac{\pi m^{2} n}{q X_{i}}}
$$

In the pure electric field $X_{i}=E$ and Eq. (25) is the Schwinger pair production rate for charged bosons.

\section{FOCK SPACE IN SCALAR QED}

In an electric field in the $z$-direction with the timedependent gauge potential $A_{\mu}=\left(0,0,0, A_{z}(t)\right)$, the Hamiltonian is given by

$$
H(t)=\int d^{3} x\left[\pi^{*} \pi+\phi^{*}\left(-\partial_{x}^{2}-\partial_{y}^{2}-\left(\partial_{z}+i q A_{z}\right)^{2}+m^{2}\right) \phi\right] \text {. }
$$

The quantum state of the charged particle obeys the functional Schrödinger equation 23]

$$
i \frac{\partial \Phi(t, \mathbf{x})}{\partial t}=H(t) \Phi(t, \mathbf{x}) \text {. }
$$

In the scattering theory the final state evolves from an initial state

$$
\left.\mid \text { out }\rangle=U\left(t_{\text {out }}, t_{\text {in }}\right) \mid \text { in }\right\rangle,
$$

where $U$ is the evolution operator

$$
i \frac{\partial U(t)}{\partial t}=H(t) U(t)
$$

The prominent feature of the Hamiltonian (26) is the explicit time-dependency through $A_{z}(t)$. For a time-dependent Hamiltonian, we may use the invariant method, in which invariant operators satisfying the Liouville-von Neumann equation provide the exact quantum states [19]. Our stratagem is to find the timedependent annihilation and creation operators for each mode of the free scalar field (26), which are invariant operators. As we now have a field instead of a finite system, we first quantize the field as

$$
\begin{aligned}
\phi(t, \mathbf{x}) & =\int[d \mathbf{k}]\left[\varphi_{\mathbf{k}}(t) a_{\mathbf{k}}(t)+\varphi_{\mathbf{k}}^{*}(t) b_{\mathbf{k}}^{\dagger}(t)\right] e^{i \mathbf{k} \cdot \mathbf{x}} \\
\phi^{*}(t, \mathbf{x}) & =\int[d \mathbf{k}]\left[\varphi_{\mathbf{k}}(t) b_{\mathbf{k}}(t)+\varphi_{\mathbf{k}}^{*}(t) a_{\mathbf{k}}^{\dagger}(t)\right] e^{-i \mathbf{k} \cdot \mathbf{x}}(30)
\end{aligned}
$$


where $[d \mathbf{k}]=d^{3} k /(2 \pi)^{3}$, and the momentum as

$$
\begin{aligned}
\pi(t, \mathbf{x}) & =\int[d \mathbf{k}]\left[\dot{\varphi}_{\mathbf{k}}^{*}(t) a_{\mathbf{k}}^{\dagger}(t)+\dot{\varphi}_{\mathbf{k}}(t) b_{\mathbf{k}}(t)\right] e^{-i \mathbf{k} \cdot \mathbf{x}}, \\
\pi^{*}(t, \mathbf{x}) & =\int[d \mathbf{k}]\left[\dot{\varphi}_{\mathbf{k}}^{*}(t) b_{\mathbf{k}}^{\dagger}(t)+\dot{\varphi}_{\mathbf{k}}(t) a_{\mathbf{k}}(t)\right] e^{i \mathbf{k} \cdot \mathbf{x}} \cdot(31)
\end{aligned}
$$

Here $a_{\mathbf{k}}$ and $a_{\mathbf{k}}^{\dagger}$ are the annihilation and creation operators for particles and $b_{\mathbf{k}}$ and $b_{\mathbf{k}}^{\dagger}$ are those for antiparticles and $\varphi_{\mathbf{k}}$ are auxiliary variables that will be determined later.

Then the Hamiltonian takes the form

$$
\begin{aligned}
H(t)= & \int[d \mathbf{k}]\left[\left(\dot{\varphi}_{\mathbf{k}}^{*} \dot{\varphi}_{\mathbf{k}}+\omega_{\mathbf{k}}^{2} \varphi_{\mathbf{k}}^{*} \varphi_{\mathbf{k}}\right)\left(a_{\mathbf{k}}^{\dagger} a_{\mathbf{k}}+b_{\mathbf{k}}^{\dagger} b_{\mathbf{k}}+1\right)\right. \\
& \left.+\left(\dot{\varphi}_{\mathbf{k}}^{* 2}+\omega_{\mathbf{k}}^{2} \varphi_{\mathbf{k}}^{* 2}\right) a_{\mathbf{k}}^{\dagger} b_{\mathbf{k}}^{\dagger}+\left(\dot{\varphi}_{\mathbf{k}}^{2}+\omega_{\mathbf{k}}^{2} \varphi_{\mathbf{k}}^{2}\right) a_{\mathbf{k}} b_{\mathbf{k}}\right]
\end{aligned}
$$

where

$$
\omega_{\mathbf{k}}^{2}(t)=\left(k_{z}+q A_{z}(t)\right)^{2}+\mathbf{k}_{\perp}^{2}+m^{2} .
$$

According to the invariant method [18, 19, 24], we require the time-dependent operators

$$
\begin{aligned}
& a_{\mathbf{k}}(t)=i\left[\varphi_{\mathbf{k}}^{*}(t) \pi_{\mathbf{k}}^{*}-\dot{\varphi}_{\mathbf{k}}^{*}(t) \phi_{\mathbf{k}}\right] \\
& a_{\mathbf{k}}^{\dagger}(t)=-i\left[\varphi_{\mathbf{k}}(t) \pi_{\mathbf{k}}-\dot{\varphi}_{\mathbf{k}}(t) \phi_{\mathbf{k}}^{*}\right]
\end{aligned}
$$

and

$$
\begin{aligned}
& b_{\mathbf{k}}(t)=i\left[\varphi_{\mathbf{k}}^{*}(t) \pi_{\mathbf{k}}-\dot{\varphi}_{\mathbf{k}}^{*}(t) \phi_{\mathbf{k}}^{*}\right], \\
& b_{\mathbf{k}}^{\dagger}(t)=-i\left[\varphi_{\mathbf{k}}(t) \pi_{\mathbf{k}}^{*}-\dot{\varphi}_{\mathbf{k}}(t) \phi_{\mathbf{k}}\right],
\end{aligned}
$$

to satisfy the Liouville-von Neumann equation

$$
\begin{gathered}
i \frac{\partial a_{\mathbf{k}}(t)}{\partial t}+\left[a_{\mathbf{k}}(t), H(t)\right]=0 \\
i \frac{\partial b_{\mathbf{k}}(t)}{\partial t}+\left[b_{\mathbf{k}}(t), H(t)\right]=0 .
\end{gathered}
$$

They are satisfied only when $\varphi_{\mathbf{k}}$ is a complex solution to the classical mode equation

$$
\ddot{\varphi}_{\mathbf{k}}+\omega_{\mathbf{k}}^{2} \varphi_{\mathbf{k}}=0 .
$$

We can fix the Wronskian conditions

$$
\dot{\varphi}_{\mathbf{k}}^{*}(t) \varphi_{\mathbf{k}}(t)-\varphi_{\mathbf{k}}^{*}(t) \dot{\varphi}_{\mathbf{k}}(t)=i,
$$

so that the standard equal-time commutation relations hold

$$
\begin{aligned}
{\left[a_{\mathbf{k}^{\prime}}(t), a_{\mathbf{k}}^{\dagger}(t)\right] } & =\delta\left(\mathbf{k}^{\prime}-\mathbf{k}\right) \\
{\left[b_{\mathbf{k}^{\prime}}(t), b_{\mathbf{k}}^{\dagger}(t)\right] } & =\delta\left(\mathbf{k}^{\prime}-\mathbf{k}\right) .
\end{aligned}
$$

The eigenstates of $a_{\mathbf{k}}$ and $b_{\mathbf{k}}$, invariant operators, are exact quantum states of the time-dependent Schrödinger equation up to time-dependent phase factors [19]. The ground state for each mode

$$
a_{\mathbf{k}}(t)\left|0_{\mathbf{k}} ; t\right\rangle=b_{\mathbf{k}}(t)\left|\overline{0}_{\mathbf{k}} ; t\right\rangle=0,
$$

is an eigenstate with zero eigenvalue. The multi-particle and antiparticle states are given by

$$
\left|n_{\mathbf{k}_{1}} \cdots ; \bar{n}_{\mathbf{k}_{2}} \cdots ; t\right\rangle=\frac{a_{\mathbf{k}_{1}}^{\dagger n_{1}}(t)}{\sqrt{n_{1} !}} \cdots \frac{b_{\mathbf{k}_{2}}^{\dagger \bar{n}_{2}}(t)}{\sqrt{\bar{n}_{2} !}} \cdots|0 ; t\rangle .
$$

It is shown in Ref. 25] that the time-dependent phase factors indeed vanish for the number states of $N_{a}=a_{\mathbf{k}}^{\dagger} a_{\mathbf{k}}$ and $N_{b}=b_{\mathbf{k}}^{\dagger} b_{\mathbf{k}}$. Thus the product of the ground states

$$
|0 ; t\rangle=\prod_{\mathbf{k}}\left|0_{\mathbf{k}} ; t\right\rangle\left|\overline{0}_{\mathbf{k}} ; t\right\rangle
$$

is the time-dependent vacuum of the field, an exact quantum state. In other words, the time-dependent vacuum is defined as

$$
a_{\mathbf{k}}(t)|0 ; t\rangle=b_{\mathbf{k}}(t)|0 ; t\rangle=0 \text {. }
$$

\section{EFFECTIVE ACTION AT ZERO TEMPERATURE}

The effective action is determined by the scattering amplitude (14) between the ingoing vacuum and the outgoing vacuum. To find the outgoing vacuum we need the Bogoliubov transformations between the annihilation operators of the ingoing vacuum and the outgoing vacuum:

$$
\begin{aligned}
& a_{\mathbf{k}, \text { in }}=\mu_{\mathbf{k}}^{*} a_{\mathbf{k}, \text { out }}-\nu_{\mathbf{k}}^{*} b_{\mathbf{k}, \text { out }}^{\dagger}, \\
& b_{\mathbf{k}, \text { in }}=\mu_{\mathbf{k}}^{*} b_{\mathbf{k}, \text { out }}-\nu_{\mathbf{k}}^{*} a_{\mathbf{k}, \text { out }}^{\dagger},
\end{aligned}
$$

where

$$
\begin{aligned}
& \mu_{\mathbf{k}}=i\left(\varphi_{\mathbf{k}}^{*}(\infty) \dot{\varphi}_{\mathbf{k}}^{\text {in }}-\dot{\varphi}_{\mathbf{k}}^{*}(\infty) \varphi_{\mathbf{k}}^{\text {in }}\right), \\
& \nu_{\mathbf{k}}=i\left(\varphi_{\mathbf{k}}^{*}(\infty) \dot{\varphi}_{\mathbf{k}}^{\text {in } *}-\dot{\varphi}_{\mathbf{k}}^{*}(\infty) \varphi_{\mathbf{k}}^{\text {in }}\right)
\end{aligned}
$$

These coefficients satisfy the relation

$$
\left|\mu_{\mathbf{k}}\right|^{2}-\left|\nu_{\mathbf{k}}\right|^{2}=1
$$

The inverse Bogoliubov transformations are

$$
\begin{aligned}
& a_{\mathbf{k}, \text { out }}=\mu_{\mathbf{k}} a_{\mathbf{k}, \text { in }}+\nu_{\mathbf{k}}^{*} b_{\mathbf{k}, \text { in }}^{\dagger}, \\
& b_{\mathbf{k}, \text { out }}=\mu_{\mathbf{k}} b_{\mathbf{k}, \text { in }}+\nu_{\mathbf{k}}^{*} a_{\mathbf{k}, \text { in }}^{\dagger} .
\end{aligned}
$$

To express the outgoing vacuum in terms of the particle states of the ingoing vacuum, we may write the Bogoliubov transformation (47) as

$$
\begin{aligned}
a_{\mathbf{k}, \text { out }} & =U\left(r_{\mathbf{k}}, \vartheta_{\mathbf{k}} ; \theta_{\mathbf{k}}\right) a_{\mathbf{k}, \text { in }} U^{\dagger}\left(r_{\mathbf{k}}, \vartheta_{\mathbf{k}} ; \theta_{\mathbf{k}}\right), \\
b_{\mathbf{k}, \text { out }} & =U\left(r_{\mathbf{k}}, \vartheta_{\mathbf{k}} ; \theta_{\mathbf{k}}\right) b_{\mathbf{k}, \text { in }} U^{\dagger}\left(r_{\mathbf{k}}, \vartheta_{\mathbf{k}} ; \theta_{\mathbf{k}}\right) .
\end{aligned}
$$

Here $U$ is the evolution operator for each mode

$$
U\left(r_{\mathbf{k}}, \vartheta_{\mathbf{k}} ; \theta_{\mathbf{k}}\right)=S\left(r_{\mathbf{k}}, \vartheta_{\mathbf{k}}\right) P\left(\theta_{\mathbf{k}}\right)
$$


where the overall phase factor and the two-mode squeeze operator are [26, 27]

$$
\begin{aligned}
P\left(\theta_{\mathbf{k}}\right)=\exp \left[i \theta_{\mathbf{k}}\left(a_{\mathbf{k}, \text { in }}^{\dagger} a_{\mathbf{k}, \text { in }}+b_{\mathbf{k}, \text { in }}^{\dagger} b_{\mathbf{k}, \text { in }}+1\right)\right] \\
S\left(r_{\mathbf{k}}, \vartheta_{\mathbf{k}}\right)=\exp \left[r _ { \mathbf { k } } \left(a_{\mathbf{k}, \text { in }} b_{\mathbf{k}, \text { in }} e^{-2 i \vartheta_{\mathbf{k}}}\right.\right. \\
\left.\left.-a_{\mathbf{k}, \text { in }}^{\dagger} b_{\mathbf{k}, \text { in }}^{\dagger} e^{2 i \vartheta_{\mathbf{k}}}\right)\right]
\end{aligned}
$$

where the squeeze parameter $r_{\mathbf{k}}$, the squeeze angle $\vartheta_{\mathbf{k}}$, and the overall phase angle $\theta_{\mathbf{k}}$ are determined by

$$
\begin{aligned}
\mu_{\mathbf{k}} & =e^{-i \theta_{\mathbf{k}}} \cosh r_{\mathbf{k}}, \\
\nu_{\mathbf{k}}^{*} & =-e^{-i \theta_{\mathbf{k}}}\left(e^{2 i \vartheta_{\mathbf{k}}} \sinh r_{\mathbf{k}}\right) .
\end{aligned}
$$

Then the outgoing vacuum is the two-mode squeezed state of the ingoing vacuum

$$
\left.\mid 0, \text { out }\rangle=\prod_{\mathbf{k}} U\left(r_{\mathbf{k}}, \vartheta_{\mathbf{k}} ; \theta_{\mathbf{k}}\right) \mid 0, \text { in }\right\rangle
$$

from which follows the scattering amplitude

$$
\langle 0, \text { out }| 0, \text { in }\rangle=\prod_{\mathbf{k}} e^{i \theta_{\mathbf{k}}}\left\langle 0, \text { in }\left|S^{\dagger}\left(r_{\mathbf{k}}, \vartheta_{\mathbf{k}}\right)\right| 0, \text { in }\right\rangle
$$

Further, the squeeze operator can be factored as [27]

$$
\begin{aligned}
S\left(r_{\mathbf{k}}, \vartheta_{\mathbf{k}}\right)= & \exp \left[\xi_{\mathbf{k}} a_{\mathbf{k}, \text { in }}^{\dagger} b_{\mathbf{k}, \text { in }}^{\dagger}\right] \\
& \times \exp \left[\frac{\gamma_{\mathbf{k}}}{2}\left(a_{\mathbf{k}, \text { in }}^{\dagger} a_{\mathbf{k}, \text { in }}+b_{\mathbf{k}, \text { in }}^{\dagger} b_{\mathbf{k}, \text { in }}+1\right)\right] \\
& \times \exp \left[-\xi_{\mathbf{k}}^{*} a_{\mathbf{k}, \text { in }} b_{\mathbf{k}, \text { in }}\right]
\end{aligned}
$$

where

$$
\begin{aligned}
\xi_{\mathbf{k}} & =-e^{2 i \vartheta_{\mathbf{k}}} \tanh r_{\mathbf{k}}, \\
\gamma_{\mathbf{k}} & =\ln \left(1-\left|\xi_{\mathbf{k}}\right|^{2}\right)=-2 \ln \left(\cosh r_{\mathbf{k}}\right)
\end{aligned}
$$

Thus the scattering amplitude is given by

$$
\langle 0, \text { out }| 0, \text { in }\rangle=\prod_{\mathbf{k}} \frac{1}{\mu_{\mathbf{k}}^{*}}
$$

Finally, we find the effective action per unit volume

$$
\mathcal{L}_{\text {eff }}=i \frac{q E}{(2 \pi)^{3}} \int d \mathbf{k}_{\perp}^{2} \ln \left(\mu_{\mathbf{k}}^{*}\right)
$$

where $q E /(2 \pi)$ is the number of states along the $z$ direction and $1 /(2 \pi)^{2}$ is the number of states for each $\mathbf{k}_{\perp}$. It also follows that the decaying amplitude of the ingoing vacuum is

$$
\mid\langle 0, \text { out }| 0, \text { in }\rangle\left.\right|^{2}=e^{-2\left(\operatorname{Im} \mathcal{S}_{\text {eff }}\right)}=e^{-2 \sum \ln \left|\mu_{\mathbf{k}}\right|},
$$

where the summation is over all possible states in momentum and spacetime.
Now we apply the formalism for the effective action to a constant electric field with $A_{z}=-E t$. In a constant electric field, the Klein-Gordon equation takes the form

$$
\left[\partial_{t}^{2}+m^{2}+\mathbf{k}_{\perp}^{2}+\left(k_{z}-q E t\right)^{2}\right] \phi_{\omega, \mathbf{k}}(t)=0 .
$$

The solution with the appropriate ingoing flux at $t=-\infty$ is

$$
\phi_{\omega, \mathbf{k}}(t)=D_{p}(z)
$$

where $D_{p}(z)$ is the parabolic cylinder function [28] and

$$
\begin{aligned}
& z=\sqrt{\frac{2}{q E}} e^{i \pi / 4}\left(k_{z}-q E t\right), \\
& p=-i \frac{m^{2}+\mathbf{k}_{\perp}^{2}}{2(q E)}-\frac{1}{2} .
\end{aligned}
$$

It has the asymptotic form $D_{p}(z) \approx e^{-z^{2} / 4} z^{p}$ for $|z| \gg 1$. In the other region at $t=\infty$, the solution takes another form [29]

$$
D_{p}(z)=e^{i p \pi} D_{p}(-z)+\frac{\sqrt{2 \pi}}{\Gamma(-p)} e^{i(p+1) \pi / 2} D_{-p-1}(-i z)
$$

From Eq. (62) we find the Bogoliubov coefficients

$$
\begin{aligned}
\mu_{\mathbf{k}} & =\frac{\sqrt{2 \pi}}{\Gamma(-p)} e^{i(p+1) \pi / 2}, \\
\nu_{\mathbf{k}} & =e^{i p \pi} .
\end{aligned}
$$

It follows that

$$
\left|\mu_{\mathbf{k}}\right|^{2}=e^{\frac{m^{2}+\mathbf{k}_{\perp}^{2}}{2 q E}}+1
$$

The effective action per unit volume and per unit time is then

$\mathcal{L}_{\mathrm{eff}}=i \frac{q E}{(2 \pi)^{3}} \int d \mathbf{k}_{\perp}^{2}\left[\ln \sqrt{2 \pi}-\ln \Gamma\left(-p^{*}\right)-i \frac{\left(p^{*}+1\right) \pi}{2}\right](65)$

From the gamma function 30]

$$
\ln \Gamma(z)=\int_{0}^{\infty}\left[\frac{e^{-z t}}{1-e^{-t}}-\frac{e^{-t}}{1-e^{-t}}+(z-1) e^{-t}\right] \frac{d t}{t},(66)
$$

after doing the contour integral over the contour of a quarter-circle in the first quadrant, we obtain

$$
\begin{aligned}
\int_{0}^{\infty} \frac{e^{-z t}}{1-e^{-t}} \frac{d t}{t}= & \frac{1}{2 i} \mathcal{P} \int_{0}^{\infty} \frac{e^{-\left(m^{2}+\mathbf{k}_{\perp}^{2}\right) s}}{\sin (q E S)} \frac{d s}{s} \\
& +\sum_{n=1}^{\infty} \frac{(-1)^{n}}{2 n} e^{-\frac{m^{2}+\mathbf{k}_{\perp}^{2}}{q E} n \pi},
\end{aligned}
$$

where $\mathcal{P}$ denotes the principal value. Thus, integrating over the momentum, the effective action is

$$
\begin{aligned}
\mathcal{L}_{\mathrm{eff}}(E)= & -\frac{q E}{16 \pi^{2}} \mathcal{P} \int_{0}^{\infty} \frac{e^{-m^{2} s}}{\sin (q E s)} \frac{d s}{s^{2}}+\cdots+ \\
& +i \frac{q E}{16 \pi^{3}} \int d \mathbf{k}_{\perp}^{2} \ln \left(1+e^{-\pi \frac{m^{2}+\mathbf{k}_{\perp}^{2}}{q E}}\right) .
\end{aligned}
$$


Removing all the possible singular terms when $E=0$, we finally obtain the effective action (21). Note that the second and third term in Eq. (66) and other terms in Eq. (65) have to removed by a regularization procedure, which may have something to do with substraction of the zero-energy and renormalization of charge. Our method recovers the standard result, which can be compared with Eq. (3.23) of Ref. [20], where the imaginary part of the effective action gives the correct pair production rate while the real part does not recover the standard form.

\section{CONCLUSION}

In this paper we advanced a Hamiltonian method to calculate the effective action in scalar QED not only in magnetic fields but also electric fields. The widely adopted heat-kernel method is simple and powerful to find the effective action of charged bosons and fermions in a constant electromagnetic field. However, it is hard to make use of it for inhomogeneous electric fields, though calculable in principle. In the new method introduced in this paper, the effective action is expressed in terms of the Bogoliubov coefficients only. It is shown how the method works for a constant electric field. Though the new method is more complicated than the heat-kernel method, it recovers correctly the effective action from the solution of the Klein-Gordon equation. It is expected that the new method may work out the effective action in inhomogeneous electric fields as well. It would be interesting to compare the effective action in inhomogeneous electric fields with the resolvent method 31] and also the derivative expansion method [32]. The effective action at finite temperature in inhomogeneous electromagnetic fields is in progress.

\section{Acknowledgments}

The authors thank Yongsung Yoon for useful comments. The work of S. P. K. was supported by the Korea Research Foundation Grant funded by the Korean Government (MOEHRD) (KRF-2007-C00167) and the work of H. K. L. was supported by the Korea Science and Engineering Foundation (KOSEF) grant funded by the Korea government (MOST) (No. R01-2006-000-10651-0).
[1] J. Schwinger, Phys. Rev. 82, 664 (1951).

[2] W. Heisenberg and H. Euler, Z. Physik 98, 714 (1936).

[3] V. Weisskopf, K. Dan. Vidensk. Selsk. Mat. Fys. Medd. XIV, 6 (1936).

[4] G. V. Dunne, "Heisenberg-Euler Effective Lagrangians: Basics and Extensions," From Fields to Strings: Circumnavigating Theoretical Physics, edited by M. Shifman, A. Vainshtein, and J. Wheater, (World Scientific, Singapore, 2005), Vol. I, pp. 445-522, hep-th/0406216.

[5] A. Ringwald, Phys. Lett. B 510, 107 (2001); "Fundamental Physics at an X-Ray Free Electron Laser," Electromagnetic Probes of Fundamental Physics, edited by W. Marciano and S. White (World Scientific, Singapore, 2003), pp. 63-74, hep-ph/0112254 "Boiling the Vacuum with an X-Ray Free Electron Laser," Quantum Aspects of Beam Physics 2003, edited by P. Chen and K. Reil (World Scientific, Singapore, 2004), pp. 149-163, hep-ph/0304139

[6] P. M. Wood and C. Thompson, "Soft Gamma Repeaters and Anomalous X-ray Pulsars: Magnetar Candiates," in Compact Stellar X-ray Sources, edited by W. H. G. Lewin and M. van der Klis (Cambridge Univ., Cambridge, 2006), pp 547-586, astrop-ph/0406133.

[7] R. C. Duncan, "Physics in Ultra-strong Magnetic Fields," astro-ph/0002442 (2000).

[8] A. K. Harding and D. Lai, Rept. Prog. Phys. 69, 2631 (2006).

[9] N. Itoh, Prog. Theor. Phys. 44, 291 (1071).

[10] A. R. Bodmer, Phys. Rev. D 4, 1601 (1971).

[11] E. Witten, Phys. Rev. D 30, 272 (1984).

[12] E. Farhi and R. L. Jaffe, Phys. Rev. D 30, 2379 (1984).

[13] C. Alcock, E. Farhi, and A. V. Olinto, Astrophys. J. 310, 261 (1986).

[14] P. Haensel, J. L. Zdunik, and R. Schaeffer, Astron. Astrophys. 160, 121 (1986).
[15] V. V. Usov, Phys. Rev. Lett. 80, 230 (1998).

[16] V. V. Usov, T. Harko, and K. S. Cheng, Astrophys. J. 620, 915 (2005).

[17] T. Harko and K. S. Cheng, Astrophys. J. 643, 318 (2006).

[18] S. P. Kim and H. K. Lee, Phys. Rev D 76, 125002 (2007).

[19] H. R. Lewis, Jr. and W. B. Riesenfeld, J. Math. Phys. 10, 1458 (1969).

[20] J. Ambjorn, R. J. Hughes, and N. K. Nielsen, Ann. Phys. 150, 92 (1983).

[21] B. G. Wybourne, Classical Groups for Physicists (A Wiley-Interscience Pub., New York, 1974), pp. 268-275.

[22] W. Greiner and J. Reinhardt, Quantum electrodynamics 2nd ed. (Springer, Berlin, 1994), pp. 376-378.

[23] K. Freese, C. T. Hill, and M. Mueller, Nucl. Phys. B 255, 693 (1995).

[24] S. P. Kim and C. H. Lee, Phys. Rev. D 62, 125020 (2000).

[25] S. P. Kim and D. N. Page, Phys. Rev. A 64, 012104 (2001).

[26] C. M. Caves and B. L. Schumaker, Phys. Rev. A 31, 3068 (1985).

[27] B. L. Schumaker and C. M. Caves, Phys. Rev. A 31, 3093 (1985).

[28] I. S. Gradshteyn and I. M. Ryzhik, Table of Integrals, Series, and Products (Academic Press, San Diego, 1994).

[29] I. S. Gradshteyn and I. M. Ryzhik, Table of Integrals, Series, and Products (Academic Press, San Diego, 1994), 9.248-3.

[30] I. S. Gradshteyn and I. M. Ryzhik, Table of Integrals, Series, and Products (Academic Press, San Diego, 1994), 8.341-3.

[31] G. Dunne and T. Hall, Phys. Rev. D 58, 105022 (1998).

[32] V. P. Gusynin and I. A. Shovkovy, J. Math. Phys. 40, 5406 (1999). 